\title{
A LONDRES: QUELQUES JALONS
}

On connaît mal la proscription anglaise. Le problème est d'abord de rassembler la documentation nécessaire, qui existe. Boris Nicolaevsky avait commencé la tâche. Les documents que nous publions ici proviennent - à l'exception de la seule lettre de Vaillant à Marx ${ }^{1}$ - des Nicolaevsky Papers conservés à la Hoover Institution de Stanford, Californie. Ils s'y trouvent en photocopies. Nous les avons consultés du vivant de Boris Nicolaevsky, à New York en 1963, et une seconde fois à Stanford, grâce à l'obligeance de $\mathrm{M}^{\mathrm{me}}$ Anna Bourguina, conservatrice de ces fonds. Ni feu Nicolaevsky ni Mme Bourguina ne purent nous renseigner sur les documents originaux dont étaient tirées ces photocopies, très anciennes en apparence. La brièveté de mes séjours ne m'a pas permis de déchiffrer tous les documents souvent peu lisibles, surtout la partie "correspondance», susceptible de fournir des précisions sur la provenance des fonds.

Voici donc le peu de renseignements que nous avons pu en tirer et que nous voudrions verser au dossier des Communards de Londres, dans l'espoir qu'ils pourront servir à des recherches plus fouillées. Il s'agit d'une documentation qui fut probablement constituée et classée par l'archiviste de la Société des réfugiés de la Commune à Londres, Albert Goullé. Mais il conviendrait mieux de parler de documents reconstitués parce que la plupart des pièces, sinon toutes, sont en effet des copies portant l'indication:

"Certifié conforme

Londres 27 septembre 1875

L'archiviste: Goullé

Les membres de la con de la Sté des Réfugiés:

Jules Oswald; Rysto"

Le problème d'identification de la source se dédouble par conséquent. Nous ne savons pas où se trouvent les pièces originales ni où sont

1 Fonds Marx-Engels, D 4356, I.I.S.G., Amsterdam. 
conservées les copies conformes établies par Goullé et ses deux camarades.

Il y a cependant quelques indications propres à guider le chercheur: 1) Une note d'une page dans la chemise $n^{0} 5$ (Pièces diverses) ${ }^{1}$ porte sur les "Archives de la Société des Réfugiés». Cette note renvoie aux procès-verbaux qui signalent le fait qu'une partie des documents avait été soustraite. Elle fournit en même temps un inventaire sommaire des procès-verbaux.

2) Dans la chemise n 2 "Catalogue, correspondance, procès-verbaux", nous trouvons une liste plus complète des séances tenues entre le 30 juillet 1871 et le 30 septembre 1875 .

3) La chemise $n^{0} 3$ est intitulée "Catalogue des pièces contenues dans ce carton dressé par Henri Granger ${ }^{2}$ les 18-23 février 1904». Dans cette chemise se trouvent en effet deux catalogues. Celui dressé par Granger en 1904 et un autre, établi probablement par l'archiviste de la Société en 1875 .

4) Dans plusieurs chemises nous trouvons des notes et des correspondances de Granger et diverses autres personnes, notamment Constant Martin, Raymond Félix et d'autres, datant des années 1880 et même 1890.

L'on peut en déduire que vers le tournant du siècle, Ernest Granger, ce fidèle ancien "lieutenant» de Blanqui, a tenté de réunir et de reconstituer ce qui restait des archives dispersées de la société défunte des réfugiés de Londres. C'est pourquoi s'y trouvent pêle-mêle trois sortes de documents: des originaux de la période 1871-1875; des «copies conformes» de la même période; des pièces diverses, postérieures, conservées par Granger.

Quant à l'authenticité des pièces recopiées - les procès-verbaux certifiés conformes par l'archiviste Goullé et deux autres membres de la Société - il en existe une preuve indirecte dans les Papiers Eudes aux Archives Nationales, Institut français d'Histoire sociale. Il s'agit d'un manuscrit de 16 pages, l'original du procès-verbal de la séance du 25 août 1872 de la Société, signé par Edouard Bourdeille, secrétaire. Le collationnement de ce document avec la copie certifiée conforme prouve en effet l'exactitude de cette dernière quant au contenu (alors que l'agencement de la copie est différent de celui de l'original). La copie, comme les autres, est marquée d'un tampon circulaire portant «Société des Réfugiés de la Commune de Paris, Fondée à Londres le 30 juillet 1871». Il n'y a pas de tampon sur l'original.

Nous avons cherché en vain d'autres indices éventuels sur ces archives dans le petit fonds Papiers Granger conservé à l'I.U.H.E.I.,

1 Pour le classement des chemises, voir Documents V ci-dessous.

2 Granger porte les prénoms de Ernest, Henri. Le premier prénom est le plus usité et c'est celui que nous employons par la suite. 
Genève, tout comme dans le fonds Eudes à l'Institut français d'Histoire sociale. En revanche Wladimir Martel, auteur de Mes entretiens avec Granger, Lieutenant de Blanqui, ${ }^{1}$ a travaillé également sur les dossiers de Granger dans diverses archives et a cité dans son ouvrage plusieurs des documents que nous avons relevés dans les Nicolaevsky Papers.

Faute d'avoir pu pousser les recherches plus loin, nous nous sommes contenté de la présentation sommaire du fonds conservé dans les Nicolaevsky Papers, ${ }^{2}$ et d'en tirer quelques documents, notamment trois lettres de Marx. Nous avons omis d'inclure des échantillons ou des extraits qui, au lieu d'apporter des éclaircissements, risquaient d'obscurcir les événements historiques du fait de leur caractère fragmentaire et isolé. Pour les mêmes raisons, toute tentative d'écrire à partir de ces documents une histoire de la Société aurait été soit un vain effort soit une entreprise beaucoup trop vaste.

La Société, comme le projet de ses statuts le montre, ${ }^{3}$ s'était constituée avant tout pour défendre les intérêts de ses membres. Plusieurs incidents prouvent que la tendance à s'abstenir des activités politiques a prévalu malgré la pression qu'exerçaient dans le sens contraire certaines autres associations. A la séance du 18 août 1872, par exemple, Longuet déclara avoir décliné l'invitation d'envoyer des délégués de la Société au Congrès de la Ligue républicaine de Londres, étant donné que la Société des réfugiés était «une société de secours et non une société politique». ${ }^{4}$ L'A.I.T., son Conseil général jusqu'à l'époque de La

1 Paris 1939.

2 Documents V ci-dessous.

3 Voir Documents $\mathrm{V}$ en annexe.

4 Les soucis financiers tenaient en fait une large place dans les réunions de la Société des Réfugiés de la Commune, si l'on en croit les rapports de police conservés aux Archives de la Préfecture de Police à Paris. Ainsi un rapport de la séance du 8 août 1871 fait état de divergences des membres de la Société avec le Conseil général de l’A.I.T. (A.P.P., B/a 434, pièces 33-34): certains réfugiés s'étaient plaints de la parcimonie avec laquelle les secours de l'A.I.T. étaient distribués. Un rapport daté du 15 août 1871 (A.P.P., B/a 434, pièces 35-37) signale que «le Conseil général se fatigue d'entendre les récriminations et les attaques que l'on dirige contre elle [l'Internationale], et si le conseil portait ces faits à la connaissance des membres anglais et des sociétés ouvrières d'Angleterre, les ressources manqueraient immédiatement aux réfugiés. Aussi ceux qui attaquent le Conseil général sont obstinément combattus par les autres..." Le mème rapport fait allusion aussi aux dissensions politiques entre les réfugiés: "L'ensemble des réfugiés étant de diverses nations et de diverses nuances politiques [et] sociales, est curieux à étudier. Les blanquistes reprochent aux socialistes modérés de n'avoir pas été assez révolutionnaires et d'avoir fait échouer le mouvement par leur modérantisme et leur administration; ces derniers reprochent aux autres d'avoir été trop violents vers la fin de la Commune alors que c'était au lendemain du 18 mars que les mesures révolutionnaires auraient dû être prises..." 
Haye et le Conseil fédéral britannique de l'Internationale par la suite, était, elle aussi, l'objet de nombreuses critiques et de suspicion selon les procès-verbaux de la Société. Les débats politiques, si on peut les appeler ainsi, avaient trait soit aux événements du passé (des controverses à propos de la Commune), soit à des intrigues et des calomnies du présent (les «affaires» des frères Caria, de Landeck, de Vésinier entre autres).

Tous ces sujets, certes, sont des reflets d'activités et de controverses politiques. Mais des reflets trop personnalisés - des déchets, est-on tenté de dire - pour servir de point de départ à une étude historique. Celle-ci devrait être entreprise sur une base infiniment plus large. Elle devrait s'étendre avant tout sur les rapports avec l'Internationale et les nombreuses sociétés politiques françaises à Londres, telles que la Commune révolutionnaire des blanquistes, ${ }^{\mathbf{1}}$ la Section française de 1871 de Vermersch, la Fédération universelle des travailleurs de Vésinier et de Landeck, ${ }^{2}$ le Comité révolutionnaire du prolétariat de V. Delahaye, ${ }^{3}$ les petits groupements comme le Cercle d'Etudes sociales de Londres ou la Société des Républicains-socialistes de Jersey. Elle devrait regrouper enfin, bien sûr, les nombreux journaux, documents, écrits et témoignages personnels des réfugiés, comme par exemple la correspondance de Jules Vallès en exil qui vient d'être présentée dans un ouvrage récent. ${ }^{4}$ Dans un tel contexte d'ensemble, les nombreuses informations sur la vie des émigrés contenues dans les archives de la Société revêtiront sans doute plus d'importance et révéleront même une nature différente, tout comme ce serait le cas des polémiques qui y sont rapportées à propos des événements de la Commune elle-même.

1 Pour deux adresses célèbres lancées depuis Londres par la Commune révolutionnaire, voir La Première Internationale, Recueil de documents publié sous la direction de Jacques Freymond, Droz, Genève (ci-après: Freymond, Recueil), t. III, pp. 138-147 ("Internationale et Révolution», 15 septembre 1872) et t. IV, pp. 385-390 ("Aux Communeux", juin 1874). Signalons que presque tous les membres de la Commune révolutionnaire étaient aussi affiliés à la Société des Réfugiés de la Commune de Londres.

2 Voir Freymond, Recueil, t. III, pp. 123-132 pour le compte rendu du congrès que la Fédération universelle des travailleurs tint à Londres en septembre 1872. ${ }_{3}$ Voir Freymond, Recueil, t. IV, pp. 110-114, pour une "Proposition" de ce comité au Congrès de Genève de l'A.I.T. (fédéraliste) de 1873.

4 Gérard Delfau, Jules Vallès, L'exil à Londres (1871-1880), Bordas, Paris 1971 (432 pp.). 


\section{DOCUMENTS}

\section{I-IV: CORRESPONDANCE MARX/VAILLANT}

Les trois lettres inédites de Marx à Vaillant et celle de Vaillant à Marx que nous présentons ici n'apportent pas de véritables révélations. Bien qu'on n'ait connu jusqu'à présent aucune lettre de Marx adressée à Vaillant, les relations étroites qu'ils entretenaient en 1871-72 ont été maintes fois relevées et documentées. ${ }^{1}$ Sur le plan politique, c'était en effet une période d'entente et de collaboration entre Marx et les blanquistes de Londres. Vaillant fut à titre personnel élu membre du Conseil général de la Première Internationale, en même temps que plusieurs autres Communards. Il prit par la suite une part extrêmement active à la Conférence de Londres de septembre 1871, de même qu'aux préparatifs et aux débats du Congrès de La Haye de 1872. Le texte de base de la fameuse résolution sur l'action politique, qui contribua de façon explosive à la scission de l'A.I.T., fut rédigé par Vaillant.

Les lettres présentes ne font donc que corroborer et illustrer des faits déjà connus. Toutefois, elles apportent quelques nuances et précisions qui méritent d'être velevées.

La brochure dont Marx annonce l'impression pour "le lundi prochain" est celle qui contient les Résolutions des délégués de la Conférence de l'Association internationale des travailleurs réunis à Londres, du 17 au 23 septembre $1871 .{ }^{2}$ Publiée au mois de novembre en 500 exemplaires, elle porte la date du 17 octobre, puisque c'est à cette séance que le Conseil général confia à Marx le soin de faire imprimer les résolutions. ${ }^{3}$

Le deuxième paragraphe du billet de Marx éclaive la façon dont la fameuse résolution $I X$ de la Conférence de Londres a trouvé sa forme définitive. A l'origine, comme nous l'avons fait remarquer, c'est Vaillant, secondé par Longuet et Martin, qui présenta à la Conférence une motion sur l'action politique. ${ }^{4}$

Les divers amendements et retouches qu'elle subit n'ont certes pas dépouillé cette proposition de son sens primitif. Mais elle fut quand même sensiblement modifiée lors des débats déjà. D'abord, Serraillier et Frankel proposèrent que le texte de Vaillant fût précédé d'un considérant, pour rappeler la fausse traduction des statuts ainsi que le fait que les statuts originaux de l'Association avaient toujours préou le caractère indissociable du "politique" et du "social". Marx appuya cet amendement qui affirmera, dit-il, "que ce n'est pas d'aujourd'hui que l'A ssociation demande que les ouvriers fassent de la politique, mais de tout temps". ${ }^{5}$ Il rappela ensuite "qu'il faut live ensemble les statuts et l'adresse inauguralen. ${ }^{6}$

1 Voir notamment Maurice Dommanget, Edouard Vaillant, un grand socialiste, 1840-1915, La Table Ronde, Paris 1956, pp. 51-58.

2 (Circulaire publiée par le Conseil général de l'Association.) Imprimerie typographique internationale, 1871 , Londres $(6 \mathrm{pp}$.$) . Reproduite dans Freymond,$ Recueil, t: II, pp. 233-239.

${ }^{3}$ The General Council of the First International 1870-1871. Minutes. Institute of Marxism Leninism, Moscou s.d. (ci-après: Minutes, t. IV), p. 306.

4 Freymond, Recueil, t. II, pp. 191 sq.

5 Ibid., p. 196.

- Ibid., p. 201. 
Un autre élément des remaniements ultérieurs apparaît également au cours des débats. Dans son intervention à la septième séance, le 21 septembre, Engels lança l'idée qui devint le véritable pivot de la résolution $I X$ sur l'action politique: "Il faut que le parti des travailleurs se constitue non pas comme s'il était à la remorque de tel ou tel parti bourgeois mais qu'il soit un parti indépendant ayant ses propres buts, sa politique propre." ${ }^{1} A$ la séance suivante, au soir du 21 septembre, Outine et Pervet présentèrent une motion portant notamment les mots "nécessité de l'action politique du parti prolétaire...» ainsi que la proposition de laisser "au Conseil général le soin de donner la rédaction finale aux deux propositions de Vaillant et de Serraillier et Frankel». Un long débat s'ensuivit au cours duquel Vaillant lui-même, entre autres, protesta contre les amendements ainsi que contre l'idé de renvoyer le texte au Conseil général. Toutefois, par une faible majorité, cette dernière idée l'emporta.

C'est ainsi que la rédaction finale de la proposition Vaillant échut au Conseil général. ${ }^{2}$ Celui-ci, à sa séance du 7 octobre 1871 présidée par Marx, délégua cette tâche à une commission composée - comme Marx le rappelle dans sa lettre - de Friedrich Engels, de Constant Martin et de Benjamin Le Moussu, ${ }^{3}$ tandis que Marx faisait partie d'une autre commission chargée, celle-ci, de la préparation de la nouvelle édition des Statuts et des résolutions de la Conférence, sous forme de circulaire. ${ }^{4}$ A la séance suivante, séance extraordinaive du 16 octobre, Engels présenta le rapport de la commission (citée indistinctement comme commission ou comité) qui donna, selon le passage précité des Minutes, "satisfaction générale" au Conseil, à l'exception d'un paragraphe ayant trait à "l'organisation militante de la classe ouvrière". Pour finir, le Conseil passa outre à cette objection et, toujours selon le même passage des Minutes, approuva le texte présenté par la commission et ordonna son impression.

C'est la lettre du 22 octobre publiée ici qui nous apprend que ce texte devait subir encore des modifications. Ayant été chargé de préparer l'impression de l'ensemble des résolutions, Marx remania du même coup l'arrangement de la résolution $I X{ }^{5}$ Nous

1 Ce passage de l'intervention d'Engels ne figure pas dans le manuscrit français original; il n'a pas été reproduit dans les procès-verbaux publiés dans Freymond, Recueil (cf. t. II, p. 197, note 31). Il a été retraduit en français d'après l'édition russe Londonskaja Konferencija pervogo internacionala 17-23.IX.1871 g., Partizdat, Moscou 1936, dans Miklós Molnár, Le Déclin de la Première Internationale, Droz, Genève 1963, pp. 218-219.

2 Freymond, Recueil, t. II, p. 205.

3 Minutes, t. IV, p. 289.

4 Ibid., pp. 289, 300 et 306.

5 Voici le texte définitif: "Vu les considérants des statuts originaux où il est dit: <L'émancipation économique des travailleurs est le grand but auquel tout mouvement politique doit être subordonné comme moyen>;

Vu l'adresse inaugurale de l'Association internationale des travailleurs (1864) qui dit: <Les seigneurs de la terre et les seigneurs du capital se serviront toujours de leurs privilèges politiques pour défendre et perpétuer leurs monopoles économiques. Bien loin de pousser à l'émancipation du travail, ils continueront à y opposer le plus d'obstacles possibles [...] La conquête du pouvoir politique est donc devenue le premier devoir de la classe ouvrière ; ; $[$.

Considérant en outre:

Que contre ce pouvoir collectif des classes possédantes le prolétariat ne peut agir comme classe qu'en se constituant lui-même en parti politique distinct, opposé à tous les anciens partis formés par les classes possédantes; 
ne connaissons pas de réponse de Vaillant à Marx à ce sujet, mais il faut croire que l'auteur du texte de base sur l'action politique finit par approuver la forme finale que Marx a donnée à la résolution. En tout cas, au Congrès de La Haye, Vaillant se prononça en faveur de l'introduction dans les Statuts d'un nouveau paragraphe, reprenant le fond de la résolution $I X$ de la Conférence de Londres, y compris la nécessité d'organiser le prolétariat en parti politique en vue de la conquête du pouvoir. ${ }^{1}$

22 oct. 71

Monsieur Vaillant,

Comme je ferai imprimer la brochure le lundi prochain, veuillez bien faire vos corrections au [?] plus vite que possible.

Quant à la résolution sur l'action politique la forme donnée d'abord par le comité (Engels, [un mot illisible, Martin?], Le Moussu) et les amendements [?] plus tard adoptés par vote [?] par le Conseil général avaient fait du tout un tel imbroglio que j'ai été forcé de changer l'arrangement.

Tout à vous, Karl Marx

Nicolaevsky Papers, photocopie

\section{II-III}

Les deux autres lettres de Marx, du 4 avril et du 24 mai 1872, confirment, elles, que les relations entre les deux personnages devaient être assez chaleureuses sur le plan non seulement politique mais aussi personnel.

Quant au livre que Marx demandait à Vaillant, il s'agit de l'ouvrage, très hostile à l'A.I.T., d'Edmond Villetard, Histoire de l'Internationale, Garnier Frères, Paris 1872. Marx en avait besoin parce qu'il était en train de préparer la première édition française, sous forme de brochure, de La Guerre civile en France, manifeste du Conseil général publié au lendemain immédiat de la Commune de Paris. Edité d'abord en anglais puis en allemand et en hollandais, ce texte parut pour la première fois en français dans L'Internationale de Bruxelles, en huit livraisons entre le 16 juillet et le 3 septembre 1871 (nos 131-138). Cette première version française (suivie d'une reproduction incomplète dans L'Egalité de Genève) a été établie d'après la seconde édition anglaise qui porte déjà la liste rectifiée des signataires. ${ }^{2}$

Que cette constitution du prolétariat en parti politique est indispensable pour assurer le triomphe de la révolution sociale et de son but suprême: l'abolition des classes;

Que la coalition des forces ouvrières déjà obtenue par les luttes économiques doit aussi servir de levier aux mains de cette classe dans sa lutte contre le pouvoir politique de ses exploiteurs.

La Conférence rappelle aux membres de l'Internationale: que dans l'état militant de la classe ouvrière, son mouvement économique et son action politique sont indissolublement unis." Freymond, Recueil, t. II, pp. 235-236.

1 Freymond, Recueil, t. II, p. 358.

2 The Civil War in France. Address of the General Council of the International Working-Men's Association. Printed and published for the Council by Edward Truelove, [Londres] 1871. Pour les différences entre les trois éditions successives de ce texte, voir les nos 29,30 et 32 du Répertoire international des sources pour 
La traduction de Villetard, mentionnée dans la lettre de Marx, constitue l'Appendice XIV (pp.327-384) de l'ouvrage du journaliste français. Elle est précédée d'une brève introduction et d'une note d'où il ressort que "cet abominable factum", comme Villetard l'appelle, a eté traduit d'après la première édition anglaise de la brochure. En effet la liste des signataires correspond à cette première édition, bien que la seconde édition anglaise, avec la liste modifiée, ait déjà vu le jour fin juin 1871 et que Villetard lui-même rapporte dans son live (pp. 272-274) les incidents qui ont amené le retrait des signatures de Lucraft et d'Odger et leur remplacement par celles de Taylor et de Roach. Apparemment Villetard ne connaissait pas le développement de l'affaire ni la seconde édition anglaise qui s'ensuivit.

En tout cas, l'édition française de La Guerre civile en France, sous forme de brochure, fut établie d'après la troisième édition anglaise. C'est pourquoi elle porte l'indication "troisième édition revue», et non pas parce qu'il s'agit, comme plusieurs historiens le croient, d'une troisième édition française. Elle parut à la mi-juin 1872. La traduction est l'ceuvre de Charles Longuet, revue et corrigée par Marx. Celui-ci, aux mois d'avril et mai 1872, c'est-à-dire à l'époque des lettres reproduites ici, s'occupait, entre autres, de la correction de la traduction de ce texte. ${ }^{1}$ Nous laissons aux spécialistes de marxologie le soin des collationnements nécessaires pour savoir s'il s'est servi de la traduction de Villetard.

4 avril 72

Mon cher Vaillant,

Je vous attends demain soir, 7 heures, pour dîner chez moi avec [nom illisible] et quelques autres amis.

Tout à vous, Karl Marx

Vous m'obligerez en me prêtant le livre de Villetard (je ne sais pas le nom) dans lequel se trouve la traduction française de notre manifeste sur la guerre civile en France.

International Working Men's Association, 256, High Holborn, London. W.C. [en-tête]

24 Mai 1872

Mon cher Vaillant

Serraillier a reçu une lettre de Bruxelles de la part de quelques proscrits là-bas qui rend nécessaire, d'après mon avis, que quelque chose soit faite ici pour éviter autant que possible tout nouveau scandal au milieu des Communards. C'est pour cela que je suis convenu avec Serraillier de prier vous (et par vous Arnaud), Cournet et Ranvier de venir demain soir (samedi, à 8 ou 9 heures, d'après votre

l'étude des mouvements sociaux aux XIXe et XXe siècles, t. II : La Première Internationale. Imprimés 1864-1876, A. Colin, Paris 1961. Notons ici seulement que la liste des signataires fut modifiée une première fois, dans la deuxième édition, à la suite des protestations d'Odger et de Lucraft et, plus tard, une seconde fois, dans la troisième édition en conséquence de l'adhésion d'un certain nombre de Communards cooptés au sein du Conseil général.

1 Lettre de Marx à Sorge, le 23 mai 1872. 
convenance) chez moi pour nous entendre sur ce qu'il y a à faire (bien entendu, le Conseil général n'a rien à faire avec l'affaire).

Tout à vous

Karl Marx

J'ai reçu une lettre de Rochat maintenant employé dans une mine dans le Borinage.

Nicolaevsky Papers, photocopies

IV

Le ton et le contenu de cette lettre témoignent clairement des intentions qui ont guidé la plume de Vaillant. Il n'est donc pas besoin de chercher plus loin pour savoir qu'il voulait, avant tout, se réconcilier avec Marx après "les événements de La Haye" qui avaient, comme on le sait, amené une tension entre eux à propos du transfert du Conseil général à New York.

Ce qui peut étonner, c'est la date. En octobre 1872, au retour du Congrès de La Haye, Vaillant était en train de préparer l'impression d'un pamphlet intitulé Internationale et Révolution. A propos du congrès de La Haye par des réfugiés de la Commune, ex-membres du Conseil général de l'Internationale, portant notamment des critiques sévères à l'adresse de l'ancien Conseil général. Cette brochure sortit des presses en novembre; nous trouvons les premiers commentaires d'Engels à son sujet dans sa lettre à Sorge du 16 novembre 1872.

Il ne pouvait donc être question d'un véritable rapprochement entre Marx et Vaillant au moment où ce dernier lui envoyait la lettre publiée ici. La seule explication de son geste semble ainsi de nature sentimentale. Tout en élargissant sa rupture politique avec Marx, Vaillant éprouvait le besoin de lui témoigner son affection personnelle. Peut-être même a-t-il agi en un moment d'hésitation, avant de s'engager dans cette polémique. En tout cas, la brouille entre les deux personnages n'était pas définitive puisque dès l'année suivante ils se sont à nouveau rencontrés à diverses occasions. ${ }^{1}$

Cher citoyen

Jeudi 10 Octobre 1872

Je suis tellement en retard avec vous que je ne sais comment m'excuser quoique je ne sois coupable ni d'indifférence ni de négligence. J'ai été tellement occupé d'affaires personnelles que je n'ai pu trouver un moment à moi depuis mon retour. Tout cela est venu se compliquer d'un déménagement et d'une installation nouvelle non encore terminée, si bien que je ne sais encore quand je pourrai aller vous voir. En attendant je tiens à vous remercier de l'envoi de la 1 ère livraison de votre ouvrage en regrettant de n'avoir pu le faire aussitôt de vive voix.

Ce que je tenais en même temps à vous dire, c'est que je ne voudrais pas que vous pussiez croire que les événements de la Haye aient laissé tracer dans mon esprit un point de vue personnel. Quoi qu'il arrive je n'oublierai jamais votre bienveillance et celle de votre famille pour moi et vous prie d'être assuré de l'estime et de la sincère affection

de votre tout dévoué E. Vaillant.

Veuillez présenter mes compliments affectueux à Madame et Mesdemoiselles Marx. Cordiale poignée de main à Longuet.

Fonds Marx-Engels D 4356, I.I.S.G.

1 Dommanget, op. cit., p. 58. 


\section{DESCRIPTION SOMMAIRE DU FONDS DE LA SOCIETE DES REFUGIES}

A l'état actuel, les documents conservés dans les Nicolaevsky Papers sont classés en deux dossiers: Procès verbaux 1871-1875 et Dossier Commune 1871.

Procès-verbaux

Le dossier des procès-verbaux n'est pas subdivisé. Il contient des copies des procèsverbaux souvent très détaillés, mais parfois aussi seulement des extraits sommaires des séances de la Société entre le 30 juillet 1871 et le 30 septembre 1875 . En tout: 263 pages in $4^{\circ}$ de diverses écritures, mais chaque séance certifiée de la façon indiquée cidessus. Plusieurs procès-verbaux portent des numéros apposés probablement ultérieurement, dont le dernier le numéro 76 . En principe, les procès-verbaux devraient en effet couvrir 76 séances entre 1871 et 1875 , d'après la liste des séances établie par l'archiviste. En raison des difficultés de la lecture, nous n'avons pu le vérifier. De toutes façons, quelques procès-verbaux de la même période se trouvent classés dans la chemise no 5 (Pièces diverses).

Commune 1871

Le dossier portant ce titre est réparti en 5 chemises:

1) Lettres

D'après l'inventaire de la chemise 2. Catalogue, correspondance, procès-verbaux, la chemise 1. Lettres devrait contenir 124 lettres des années 1871-1876. En fait, il y en a beaucoup moins et, comme nous l'avons déjà indiqué, elles sont mélangées avec la correspondance, postérieure à cette date, de Granger. En dehors des lettres de Marx présentées ci-dessus, signalons-en quelques autres:

Une lettre de Wilhelm Liebknecht à Vaillant (date du timbre postal: 7 août 1871) qui télicite ce dernier d'avoir "échappé aux assassins de Versailles". Il lui demande par la même occasion d'écrire une histoire succincte de la Commune pour le Volksstaat et de procurer des documents à Liebknecht pour qu'il puisse écrive l'histoire de la Commune. ${ }^{1}$

Copie d'une lettre de Gustave Maroteau à Flotte, écrite en prison, le 14 juin 1872.

Copie, certifiée conforme par Constant Martin, d'une lettre de Garreau à sa femme, écrite dans la prison de Mazas, le 26 mai 1871. Pendant la Commune, Maurice Garreau etait le directeur de la prison de Mazas. La veille de la prise de la prison par les Versaillais, il reçut "l'ordve du comité de salut public de préparev l'incendie de Mazas". "Je répondis par écrit, poursuit la lettre, que je ne le ferai pas par humanité pour les femmes et les enfants qui étaient restés à l'intérieur." D'après sa lettre, Garreau demanda même l'ordre officiel d'élargissement des prisonniers. Finalement, les prisonniers furent libérés et Garreau vetenu par les anciens employés de la prison jusqu'à l'arrivée des troupes de Versailles qui le fusillèrent quelques heures après.

Plusieurs lettres portent sur les aspects les plus divers de la vie des réfugiés à Londres. Des demandes d'aide, de logis, d'emploi. Il y a aussi un appel aux ouvriers anglais afin qu'ils acceptent leurs camarades trançais comme "des frères et non [comme] des concurrents". L'appel, ${ }^{2}$ sans date, est signé par J.-B. Clément, rappor-

1 La réponse de Vaillant à cette lettre, du 18 août 1871 , est conservée à l'I.I.S.G. d'Amsterdam (Fonds Liebknecht, L 325/1-2). Vaillant y apprend à Liebknecht qu'une riche collection de documents avait été brûlée par la personne à qui il l'avait confiée, car elle avait cédé «à l'impression de terreur générale". "C'est pour moi un véritable désastre et j'en suis maintenant d'autant plus désolé que j'espérais pouvoir vous aider dans votre projet que j'approuve fortement d'écrire l'histoire de la Commune de Paris."

2 La deuxième partie en a été reproduite par Wladimir Martel, op. cit., pp. 86-87. 
teur de la Commission de travail composée des représentants d'une douzaine de métiers. Une lettre d'Octave Caria adressée à "Mon vieux complice" rapporte l'une des séances houleuses de la Société des Réfugiés.

2) Catalogue, correspondance, procès-verbaux

En fait deux inventaires se trouvent seulement dans cette chemise:

La liste des séances et des documents annexes du 30 juillet 1871 au 30 septembre 1875. Cet inventaire est établi par les mêmes personnes, Goullé, Oswald et Rysto, qui firent les copies des procès-verbaux et en certifièrent l'exactitude.

L'autre inventaire porte en principe sur la correspondance classée dans la chemise 1, mais d'où manque un grand nombre des 124 lettres énumérées dans la liste.

3) Catalogue des pièces contenues dans ce carton dressé par Henri Granger les 18-23 février 1904

Comme nous l'avons déjà indiqué, il s'agit ici d'un catalogue établi par Granger en vue de reclasser les papiers de la Société selon un système de classement qui lui est propre. Seuls le dépouillement complet des documents et leur recoupement avec tous les catalogues auraient permis d'établir l'état actuel des fonds et de repérer les pièces perdues.

4) Liste des membres de la Société des Réfugiés

Liste de 10 pages (page de titre incluse) établie d'après les registres de la Société. Elle porte environ 500 noms avec l'indication de la profession et la date d'admission de la plupart des membres. La rubrique Observation indique souvent d'autres faits: démission, expulsion, etc. Cette liste est toutefois trop peu lisible pour que nous la reproduisions ici.

5) Pièces diverses

Chemise sans titre avec contenu divers: lettres, manifestes, statuts, etc. Nous en avons déjà cité une pièce d'une page intitulée Archives de la Société des Réfugiés. En outre, il s'y trouve notamment une Liste des réfugiés de la Commune à Londres, le 10 mai 1874. La comparaison de cette pièce de deux pages avec la Liste des membres indiquée plus haut permet de constater la forte diminution des effectifs de la Société. Nous y trouvons environ une centaine de noms de membres actifs et une cinquantaine d'expulsés, radiés ou démissionnaires, contre environ 500 au total dans la liste précédente. Fait qui donne à croire que la Société en dépérissement a tout simplement perdu de vue la majorité de ses membres.

Nous avons relevé enfin deux documents que nous reproduisons integralement ci-après à cause de leur importance en tant que premières pièces constitutives de la Société, ses Statuts ainsi que le Règlement de 1875.

Statuts (projet de contrat)

votés dans la séance du 5 août 1871

Assemblée des Réfugiés de la Commune

L'Assemblée des Réfugiés de la Commune déclare s'en remettre pour la gestion de ses intérêts aux règles suivantes:

art. 1. - L'Assemblée se réunira tous les dimanches à 5 heures dans un local déterminé.

art. 2. - Elle délègue pour la représenter dans son administration un comité de 9 membres auquel s'ajouteront a partir des 100 premiers inscrits à chaque nouveau groupe de 25 réfugiés 3 membres choisis par [parmi?] toute l'Assemblée par [pour?] les arrivants.

art. 3. - Le comité est élu pour 3 mois et renouvelable par tiers à la dernière séance de chaque mois. Le premier [mot illisible] le second, à réélire après le $1^{\mathrm{er}}$ mois et le second sera tiré au sort. Les membres sortants sont rééligibles. 
art. 4. - Le comité désignera son président de séance, son secrétaire et ses trésoriers.

art. 5. - Le comité s'entendra avec les associations [?] et les personnes qui voudront aider à l'œuvre. Il recevra les dons et les distribuera.

art. 6. - Les secours en argent seront remis chaque semaine individuellement. Tout réfugié a le droit de donner une délégation à un autre membre de l'assemblée pour toucher à sa place.

art. 7. - Une somme sera réservée chaque semaine pour subvenir aux besoins urgents.

art. 8. - Le Comité dressera la liste des réfugiés avec leurs noms, leurs adresses [deux lignes illisibles] les cas particuliers qui devront être soumis à l'assemblée. art. 9. - A chaque réunion hebdomadaire, le comité présentera un rapport sur sa gestion.

art. 10. - Le comité pourra en cas urgent convoquer l'assemblée en faisant connaître l'objet de la réunion. Le quart des membres de l'assemblée pourra également user de ce droit.

art. 11. - Toutes les dépenses devront être autorisées par l'assemblée.

art. 12. - Les votes de l'assemblée réunie régulièrement seront toujours valables. art. 13. - L'assemblée nommera à chaque réunion un président, un assesseur et un secrétaire qui feront office de bureau. [suite illisible]

art. 14. - Les présents Statuts ne peuvent être révisés qu'en assemblée extraordinaire réunissant au moins les deux tiers des [membres?] et à la majorité des trois quarts des votants.

En cas de nombre insuffisant à une première réunion, une seconde sera convoquée et votera à la majorité des trois quarts des membres présents quel qu'en soit le nombre.

Le vote sera nominal.

Certifié conforme

Certifié conforme

Londres, 277 bre 1875

Granger B. Sachs.

Archiviste Goullé

Les membres de la Con

de la Société des Réfugiés

Oswald Rysto

Société des Réfugiés de la Commune de Paris

La Société des Réfugiés de la Commune de Paris a pour but d'établir des liens de solidarité entre les combattants de la Commune à Londres.

\section{Règlement}

I Ie siège de la Société est à Londres.

II Pour être admis à faire partie de la Société, il suffit [mots illisibles $]^{11}$ la Commune de Paris.

III Les proscrits des mouvements révolutionnaires qui se sont [une ligne illisible] province, à l'appui de la Commune de Paris, sont admis à faire partie de la Société.

IV La qualité de proscrit sera établie par deux présentateurs, qui feront reconnaître les titres du candidat.

1 Entendre naturellement: d'avoir participé à / milité dans. 
V Le vote sur l'admission aura lieu dans la séance de la présentation, à la majorité des citoyens présents. Cette admission est essentiellement provisoire et ne devient définitive qu'à la séance suivante. La Commission devra à cette séance déposer son rapport sur l'admission.

VI Nulle présentation ne pourra être faite sans qu'il en ait été préalablement donné avis à la Commission.

VII La Société se réunit le dernier jeudi de chaque mois, à l'ouverture de la séance elle forme son bureau en nommant un président, un assesseur et un secrétaire.

VIII Il est nommé une Commission d'administration composée de trois membres. Elle est renouvelée par tiers tous les mois.

IX La Commission choisit dans son sein un trésorier et un secrétaire.

$\mathrm{X}$ La Commission est chargée des enquêtes sur les admissions, de l'administration de la Société. Elle envoie les feuilles de collectes, perçoit les cotisations, distribue les secours. Elle prépare les ordres du jour des séances de la Société. Elle prend, sous sa responsabilité, toutes les mesures qu'elle juge nécessaires dans l'intérêt de la Société.

XI En cas d'urgence, elle convoque les membres de la Société en assemblée générale extraordinaire.

XII A chaque séance de la Société, le secrétaire donne lecture de la correspondance, le trésorier rend compte des opérations de la Société pendant le mois écoulé, qui sont soumises à l'approbation de la Société en assemblée.

XIII I a Commission tient ses séances chaque dimanche, à neuf heures du matin. Elle doit, en outre, se réunir dans le local des séances de la Société, une heure avant l'ouverture de la séance.

$\mathrm{XIV}$ Il est nommé, tous les trois mois, en même temps que la Commission d'administration, une Commission de contrôle composée de trois membres.

$\mathrm{XV}$ Cette Commission devra se réunir pour vérifier les comptes le dimanche qui précédera chaque assemblée générale.

XVI La Commission d'administration tient un registre des adresses des membres de la Société.

XVII La Société nomme un archiviste chargé de conserver tous les documents de la Société, de les tenir en bon ordre et d'en mettre au courant un catalogue exact.

XVIII Les membres des Commissions sont rééligibles.

XIX La cotisation est fixée à 6 pence par semaine. Elle est perçue au moyen des feuilles de collecte délivrées à des collecteurs choisis par la Commission d'administration.

XX Tout membre reste débiteur envers la Société de l'arriéré de ses cotisations.

XXI Les citoyens qui ne pourraient être ni collecteurs ni collectés devront faire parvenir leurs cotisations par versement mensuel.

XXII Tout membre qui pendant six mois n'aura pas fait acte de sociétaire pourra être rayé des contrôles de la Société.

XXIII Nulle accusation contre un membre de la Société ne pourra être portée devant l'Assemblée sans avoir été, au préalable, présentée à la Commis-sion d'administration qui fera un rapport à l'Assemblée si elle le juge nécessaire.

XXIV L'Assemblée procédera, par mesure disciplinaire, à la radiation ou à l'expulsion des membres qu'elle jugera ne devoir plus faire partie de 
la Société.

XXV Les démissions devront être présentées à la Commission. L'Assemblée votera sur les conclusions de la Commission.

XXVI Le vote sur les démissions aura lieu à main levée. Pour les radiations et les expulsions, il sera procédé à l'appel nominal.

XXVII Tous les votes de l'Assemblée auront lieu à la majorité des membres présents.

Fait et approuvé en Assemblée générale le jeudi 29 avril 1875.

Le secrétaire

le rapporteur

A. Giraud

E. Durmoy 